\title{
Two particle spectrum of tensor multiplets coupled to $\mathrm{AdS}_{3} \times S^{3}$ gravity
}

\author{
Francesco Aprile®* \\ Dipartimento di Fisica, Università di Milano-Bicocca \& INFN, \\ Sezione di Milano-Bicocca, I-20126 Milano, Italy \\ Michele Santagata $\odot^{\dagger}$ \\ School of Physics and Astronomy, University of Southampton, Highfield SO17 1BJ, United Kingdom
}

(Received 24 May 2021; accepted 5 December 2021; published 23 December 2021)

\begin{abstract}
We study certain infinite families of two-particle operators exchanged in $4 \mathrm{pt}$ correlators $\left\langle\mathcal{O}_{p_{1}} \mathcal{O}_{p_{2}} \mathcal{O}_{p_{3}} \mathcal{O}_{p_{4}}\right\rangle$ of tensor multiplets living on the $\mathrm{AdS}_{3} \times S^{3}$ background. This is the weakly curved, weakly coupled SUGRA theory dual to the D1-D5 system with RR flux. At tree level in Mellin space, all these correlators are nicely determined by a single amplitude, which makes manifest the large $p$ limit, the connection with the flat space S-matrix, and a six dimensional conformal symmetry. We compute the $(1,1) \times \overline{(1,1)}$ superconformal blocks for the two-dimensional $\mathcal{N}=(4,4)$ conformal theory at the boundary, and then we obtain a formula for the anomalous dimensions of the two-particle operators exchanged in the symmetric and antisymmetric flavor channels. These anomalous dimensions solve a mixing problem which is analogous to the one in $\mathrm{AdS}_{5} \times S^{5}$ with interesting modifications. Along the way we show how the $(1,1) \times \overline{(1,1)}$ superconformal blocks relate to those in $\mathcal{N}=4 \mathrm{SYM}$ in four dimensions, and provide new intuition on the known data for $\mathrm{AdS}_{5} \times S^{5}$.
\end{abstract}

DOI: 10.1103/PhysRevD.104.126022

\section{INTRODUCTION}

Understanding what are the possible UV completions of classical gravity is one of the most exciting and challenging problems of modern theoretical physics. Valuable help might come from solving the same problem, but in spaces with an AdS factor, where the AdS/CFT correspondence plays an important role [1]. In such circumstances, would a low energy field theorist be able to reconstruct the underlying curved string theory, let us say, out of scattering data of gravitons and single particles operators? and how?.

Recent work, based on analytic bootstrap techniques in the dual conformal field theory (CFT), has shown how to address this question in $\mathrm{AdS}_{5} \times S^{5}$ SUGRA, away from the classical regime [2-10] and up to one-loop in Newton's constant. Most notably, the simplest one-loop amplitude for four gravitons (supermultiplets) was computed in [4] by implementing a bootstrap program which, in order to determine the amplitude, used the self-consistently of the operator product expansion (OPE) for all spins. The main

\footnotetext{
francesco.aprile1@unimib.it

†.Santagata@soton.ac.uk
}

Published by the American Physical Society under the terms of the Creative Commons Attribution 4.0 International license. Further distribution of this work must maintain attribution to the author(s) and the published article's title, journal citation, and DOI. Funded by SCOAP . observation in [4] was to notice that when scattering states are single particle operators, such as gravitons and KaluzaKlein modes, the operators flowing in the OPE with leading order CFT data are two-particle operators, and the latter can be studied by solving a well-defined mixing problem at tree level [11]. The CFT data of the two-particle operator can then be used to construct the leading discontinuities of the one-loop correlator and, with the help of crossing symmetry, bootstrap the full correlator.

Even though the two-particle bootstrap does not completely fix the amplitude, the left over ambiguities were shown to be very constrained, with finite spin support. ${ }^{1}$ Interpreted as an effective field theory computation, this result encourages the idea that the presence of ambiguities, at least in some theories of gravity, might not be as severe as naive considerations would suggest. In fact, the same twoparticle bootstrap program was then extended to compute one-loop 4pt amplitudes of arbitrary external single particle operators, carrying Kaluza-Klein charge under the sphere. The one-loop 4pt amplitudes so constructed were shown to pass spectacular consistency checks [8].

With analogous surprise, the spectrum of anomalous dimensions of two-particle operators in $\mathrm{AdS}_{5} \times S^{5}$ is not completely lifted by tree level supergravity $[11,14]$, but

\footnotetext{
${ }^{1}$ In the case of four gravitons (supermultiplets), the only spin zero ambiguity was resolved independently in $[12,13]$.
} 
remains partially degenerate. Only $\alpha^{\prime}$ corrections lift this partial degeneracy uniquely [15-17], despite the fact that the curved Virasoro-Shapiro amplitude [18] is not uniquely determined within the bootstrap approach, because of ambiguities similar to those mentioned previously. Again, we seem to converge on the concrete possibility that a rich mathematical structure lies within $\operatorname{AdS}_{d+1} \times$ $S^{d+1}$ supergravity, as for the beautiful hidden conformal symmetry discovered in [19].

In this paper we will continue exploring the structure of $\mathrm{AdS}_{d+1} \times S^{d+1}$ gravity, by studying the case of $\mathrm{AdS}_{3} \times S^{3}$. In particular, by studying tree level amplitudes of (chiral primaries) single particle fields in the weakly curved SUGRA regime of the D1-D5 system with RamondRamond fluxes [20-22]. Currently, this is the only other SUGRA background in which the spectrum of the dual (strongly coupled) CFT theory can be investigated in great detail with our method. In fact, we will proceed in parallel with $\mathrm{AdS}_{5} \times S^{5}$, first by developing the necessary superconformal block technology, with $\mathcal{N}=(4,4)$ superconformal symmetry, and then use it to extract OPE data from the tree level correlators $\left\langle\mathcal{O}_{p_{1}} \mathcal{O}_{p_{2}} \mathcal{O}_{p_{3}} \mathcal{O}_{p_{4}}\right\rangle$ bootstrapped in [23-27].

The single particle operators $\mathcal{O}_{p}$ living on $\mathrm{AdS}_{3} \times S^{3}$ originate from a number $n$ of tensor multiplets in the $6 \mathrm{~d}$ supergravity compactified on $S^{3}$, denoted by $s^{I}$ in [28-31], and therefore have also a flavor index, besides the KaluzaKlein charge $p$. Following the unmixing approach of $[11,14]$, we will compute tree level anomalous dimensions of certain two-particle operators with flavor, denoted afterwards by $\mathcal{O}_{(r s)}^{+}$, exchanged in $\left\langle\mathcal{O}_{p_{1}} \mathcal{O}_{p_{2}} \mathcal{O}_{p_{3}} \mathcal{O}_{p_{4}}\right\rangle$. We will find a very simple answer, which, apart for the factor of $\mathbb{D}$ explained in due course, takes the following form,

$$
\begin{aligned}
\eta_{\tau, l,[a b]}^{+}(r s) & =-\frac{2}{N} \frac{\mathbb{D}_{\tau, l,[a b]}}{\left(\ell_{6 d}+1\right)_{2}} \\
\ell_{6 d} & =l+2 r-a-1-\frac{1+(-1)^{a+l}}{2}
\end{aligned}
$$

where the $\mathrm{AdS}_{3}$ quantum numbers $\tau, l$ are the free theory dimension and spin, then $[a b]$ are the $S^{3}$ quantum numbers, and finally $(r s)$ is a pair of integers indexing the twoparticle operator. Remarkably, $\ell_{6 d}$ is essentially the only quantity controlling the anomalous dimensions, and it has the interpretation of a $6 \mathrm{~d}$ effective spin. In particular, it only depends on $r$, rather than $(r s)$. Thus, similarly to what happens in $\operatorname{AdS}_{5} \times S^{5}$, anomalous dimension with a structure like (1) are degenerate as long as the $\mathrm{AdS}_{3} \times S^{3}$ quantum numbers give the same value for $\ell_{6 d}$.

The tensor multiplet correlators $\left\langle\mathcal{O}_{p_{1}} \mathcal{O}_{p_{2}} \mathcal{O}_{p_{3}} \mathcal{O}_{p_{4}}\right\rangle$ on $\mathrm{AdS}_{3} \times S^{3}$ in fact benefit from a hidden $6 \mathrm{~d}$ conformal symmetry, which nicely resum them all into a single Mellin amplitude, and simply explains the residual degeneracy of the two-particle spectrum in (1). In $\mathrm{AdS}_{5} \times S^{5}$ this is a 10d conformal symmetry [19]. What is intriguing about this parallelism is the way the hidden conformal symmetry actually goes across dimensions. We will show indeed that, as a byproduct of our studies here, superconformal blocks for both $\mathrm{AdS}_{5} \times S^{5}$ and $\mathrm{AdS}_{3} \times S^{3}$ can be treated at once by using the $(1,1) \times \overline{(1,1)}$ formalism that we will introduce.

\section{TREE LEVEL CORRELATORS}

The correlators we are interested in have a (generalized) disconnected free part and a dynamical contribution of the form

$$
\left\langle\mathcal{O}_{p_{1}} \mathcal{O}_{p_{1}} \mathcal{O}_{p_{1}} \mathcal{O}_{p_{4}}\right\rangle_{\text {dyn. }}=\text { kinematics } \times \mathcal{A}_{\vec{p}}
$$

where $\mathcal{A}$ will denote the amplitude of the correlator. We will clarify in the next section what kinematics stands for, and what are all the allowed superconformal structures, such that $\mathcal{A}$ is function of the cross ratios $U, V$ in spacetime, and $\tilde{U}, \tilde{V}$ on the sphere [32]. Equation (2) is a non perturbative statement, and the $\mathrm{AdS}_{3} \times S^{3}$ amplitude we will study fits (2) for a specific choice of kinemat ics, hereafter denoted by $\mathcal{A}_{\vec{p}}^{+}$.

Parametrising spacetime insertion points with $4 d$ embedding coordinates, these are given by

$$
U=\frac{X_{12} X_{34}}{X_{13} X_{24}} ; \quad V=\frac{X_{14} X_{23}}{X_{13} X_{24}}
$$

with $X_{i j}=X_{i} \cdot X_{j}$ and $X_{i i}=0$ for $i, j=1,2,3,4$. Similarly, $\tilde{U}$ and $\tilde{V}$ are defined as above with the replacement $X \rightarrow Y$, where $Y_{i}$ are null and parametrize internal space insertion points. Then, the natural language to write $\mathcal{A}_{\vec{p}}^{+}$is Mellin space [34], upgraded for $\operatorname{AdS}_{d+1} \times S^{d+1}$ backgrounds as done in [33]. Indeed, the following very compact representation holds for $\mathcal{A}_{\vec{p}}^{+}$,

$\mathcal{A}_{\vec{p}}^{+}=-\oint d s d t \sum_{\tilde{s}, \tilde{t}, \tilde{u}} U^{s} V^{t} \tilde{U}^{\tilde{s}} \tilde{V}^{\tilde{t}}\left(\Gamma_{\otimes} \times \mathcal{M}_{\vec{p}}(s, \tilde{s}, \ldots)\right)$

where

$$
\begin{aligned}
\Gamma_{\otimes}= & \frac{\Gamma[-s] \Gamma\left[-s+c_{s}\right]}{\Gamma[1+\tilde{s}] \Gamma\left[1+\tilde{s}+c_{s}\right]} \times \frac{t-\text { channel }}{\tilde{t}-\text { channel }} \\
& \times \frac{u-\text { channel }}{\tilde{u}-\text { channel }}
\end{aligned}
$$

with $^{2}$

\footnotetext{
${ }^{2}$ That $p_{3}$ appears singled out is conventional, and will have to do with kinematics. A trivial change of variables can be used to implement permutation symmetric conventions, but there is no point in doing that since superconformal blocks do not have such a symmetry. In fact, in both cases another change of variables to $\{\mathbf{s}, \tilde{\mathbf{s}}, \ldots\}$ is needed to manifest crossing. See Eq. (2.12) of [17].
} 


$$
\begin{aligned}
s+t+u & =-p_{3}-1 ; \quad \tilde{s}+\tilde{t}+\tilde{u}=p_{3}-1 \\
c_{s} & =\frac{p_{1}+p_{2}-p_{3}-p_{4}}{2} ; \quad c_{t}=\frac{p_{1}+p_{4}-p_{2}-p_{3}}{2} ; \quad c_{u}=\frac{p_{2}+p_{4}-p_{3}-p_{1}}{2}
\end{aligned}
$$

Here $s, t, u$ and $\tilde{s}, \tilde{t}, \tilde{u}$ are a choice of Mellin variables, and the triplet $c_{s}, c_{t}, c_{u}$ accompanies our choice in charge space. Note that the sum (4) is restricted to the triangle $\tilde{s} \geq-\min \left(0, c_{s}\right), \tilde{t} \geq-\min \left(0, c_{t}\right), \tilde{u} \geq-\min \left(0, c_{u}\right)$ due to the $\Gamma$ function in the denominator of $\Gamma_{\otimes}$. It can also be turned into a contour integral. This is useful since it was shown in [33] that upon taking $p_{i}$ large the integrals localize on a classical saddle point whose action is determined just by $\Gamma_{\otimes}$. The result matches the computation of four geodesics shooting from the boundary and meeting in the bulk. Reading off the momenta at the intersection point, it was understood that the combinations of Mellin variables

$$
\mathbf{s}=s+\tilde{s} ; \quad \mathbf{t}=t+\tilde{t} ; \quad \mathbf{s}+\mathbf{t}+\mathbf{u}=-2
$$

evaluated at the saddle point, become proportional to actual Mandelstam invariants of a flat space scattering process in higher dimensions, where the sphere is decompactified. This nicely explains that $\lim _{p \rightarrow \infty} \mathcal{M}$ is fixed by the flat space $S$-matrix $[35,36]$ and provides a canonical covariantization of $\mathcal{M}_{1111} \rightarrow \mathcal{M}_{\vec{p}}$ onto $\operatorname{AdS}_{3} \times S^{3}$, yielding the result

$$
\mathcal{M}_{1111}(\mathbf{s}, \mathbf{t})=\frac{\delta^{12} \delta^{34}}{\mathbf{s}+1}+\frac{\delta^{14} \delta^{23}}{\mathbf{t}+1}+\frac{\delta^{13} \delta^{24}}{\mathbf{u}+1}
$$

where the $\delta^{i j} \equiv \delta^{I_{i} I_{j}}$ are $n$ dimensional Kronecker deltas referred to the flavor indexes $I$ that we have been omitting until now. Upon assuming the existence of a $6 \mathrm{~d}$ conformal symmetry, (8) becomes the exact result,

$$
\mathcal{M}_{\vec{p}}=\mathcal{M}_{1111}(\mathbf{s}, \mathbf{t}) .
$$

In particular, we can read off the Mellin amplitude for generic charges $\vec{p}$ out of the very same $\mathcal{M}_{1111}$.

The flavor structure of the correlators will be decomposed in channels, e.g., [37,38]. Thus, we introduce the singlet, $\mathbb{I}$, the symmetric, $\mathbb{S}$, and the antisymmetric channel, A. In the order,

$$
\begin{aligned}
& \mathcal{M}_{\vec{p}}^{\mathbb{I}}=\frac{1}{n}\left[\frac{1}{\mathbf{t}+1}+\frac{1}{\mathbf{u}+1}\right]+\frac{1}{\mathbf{s}+1} ; \quad \mathbb{I}=\delta^{12} \delta^{34} \\
& \mathcal{M}_{\vec{p}}^{\mathbb{S}}=\frac{1}{2}\left[\frac{1}{\mathbf{t}+1}+\frac{1}{\mathbf{u}+1}\right] ; \quad \mathbb{S}=\delta^{13} \delta^{24}+\delta^{14} \delta^{23}-\frac{2}{n} \delta^{12} \delta^{34} \\
& \mathcal{M}_{\vec{p}}^{\mathbb{A}}=\frac{1}{2}\left[\frac{1}{\mathbf{t}+1}-\frac{1}{\mathbf{u}+1}\right] ; \quad \mathbb{A}=\delta^{14} \delta^{23}-\delta^{13} \delta^{24}
\end{aligned}
$$

We will focus on $\mathcal{M}_{\vec{p}}^{f}$ with $f=\mathbb{S}, \mathbb{A}$, since these are closed sectors. For the singlet channel one should include other correlators in the $6 \mathrm{~d}(2,0)$ supergravity [25,27], such that all allowed two-particle operators participate.

\section{SUPERCONFORMAL SYMMETRY}

The dual conformal field theory that we are studying has $\mathcal{N}=(4,4)$ superconformal symmetry in $2 \mathrm{~d}$, and the relevant superconformal blocks belong to the product $(1,1) \times \overline{(1,1)}$, where the notation $(1,1)$ refers to superconformal blocks of $S U(1,1 \mid 2)$, studied in [39]. These superconformal blocks are labelled by a Young diagram $\underline{\kappa}=\left[\kappa, 1^{\kappa^{\prime}-1}\right]$ with at most one row and one column, of length $\kappa$ and $\kappa^{\prime}$ respectively, thus $(1,1)$. In fact, Heslop and Doobary wrote in [39] a beautiful determinantal formula for the more general $(m, n)$ superconformal blocks. Borrowing that result, we introduce the $(1,1)$ superconformal block

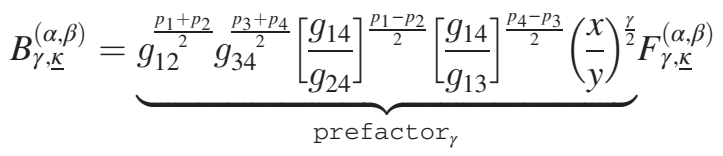

where $\alpha=\max$ and $\beta=\min \left(\frac{\gamma-p_{12}}{2}, \frac{\gamma-p_{43}}{2}\right)$,

$$
F_{\gamma, \underline{\underline{K}}}^{(\alpha, \beta)}=\delta_{\underline{\kappa}, \underline{0}}\left(\frac{y}{x}\right)^{\beta}+(x-y) H_{\underline{\kappa}}(x, y)
$$

and the dependence on $\underline{\kappa}$ enter through

$H_{\underline{\kappa}}= \begin{cases}\sum_{k=0}^{\beta-1} h_{-k}^{(\alpha, \beta, \gamma)}(x) h_{k+1}^{(-\alpha,-\beta,-\gamma)}(y) & \underline{\kappa}=\underline{0} \\ (-)^{\kappa^{\prime}-1} h_{\kappa}^{(\alpha, \beta, \gamma)}(x) h_{\kappa^{\prime}}^{(-\alpha,-\beta,-\gamma)}(y) & \text { otherwise }\end{cases}$

with $h_{k}^{(a, b, c)}(z)=z^{k-1}{ }_{2} F_{1}(k+a, k+b ; 2 k+c ; z)$. The parameter $\gamma$ specifies, together with the Young diagram $\underline{\kappa}$, the exchanged representation. It plays an important role for short representations. However, since we will be mainly interested in long representation, it will not be essential in our discussion. It is nevertheless useful to understand his origin diagrammatically, for example in free theory. Indeed, as it appears in the prefactor in (11), it counts the powers of cross ratios $x / y=\left(g_{13} g_{24}\right) /\left(g_{12} g_{34}\right)$, and therefore the number of propagators going from points (12) to (34), so we can think of it as setting the origin for the dimension of the exchanged operators in that diagram.

Note that $F_{\gamma, \underline{K}}$ has a polynomial expansion in both $x$ and $y$. In particular, $F_{\gamma, \varnothing}=1+\cdots$, since the resummation in $x$ 
is nontrivial whenever $\beta \geq 1$. Note also that $\kappa^{\prime} \leq \beta$, otherwise $F_{\gamma, \underline{\underline{K}}}$ vanishes (in the $y$ ). In fact the $F_{\gamma, \underline{\underline{K}}}$ is better defined as an expansion over super Schur polynomials of the form

$$
F_{\gamma, \underline{\underline{K}}}=\sum_{\underline{\underline{\nu}: \underline{\underline{\underline{ }}} \underline{\underline{L}} \underline{\underline{L}}}}\left(T_{\gamma}\right) \underline{\underline{\underline{\kappa}}} s_{\underline{\underline{\nu}}}(x \mid y)
$$

where, by construction, $s_{\underline{\nu}}(x \mid y)$ and their multivariate generalization, solve the superconformal Ward identity,

$$
\left[\left(\partial_{x_{i}}+\partial_{y_{j}}\right) s_{\underline{\underline{\nu}}}\right]_{x_{i}=y_{j}}=0
$$

and thus the superconformal block so constructed.

A basis for the $\mathcal{N}=(4,4)$ superconformal blocks is obtained by taking products of such $F$. On the real slice, we will distinguish among,

\begin{tabular}{|c|c|}
\hline$B_{\varnothing}(x, y) B_{\varnothing}(\bar{x}, \bar{y})$ & half-BPS \\
\hline$B_{\underline{\underline{\kappa}}}(x, y) B_{\varnothing}(\bar{x}, \bar{y})+$ c.c. & short \\
\hline$B_{\underline{\kappa}_{1}}(x, y) B_{\underline{\kappa}_{2}}(\bar{x}, \bar{y})+$ c.c. & long \\
\hline
\end{tabular}

In each of these cases the result always fits into the form

$\mathcal{G}=\mathcal{C}+[(x-y) \mathcal{S}(x, y)+$ c.c. $]+(x-y)(\bar{x}-\bar{y}) \mathcal{H}(x, \bar{x}, y, \bar{y})$

where $\mathcal{C}$ is a constant, while $\mathcal{S}$ and $\mathcal{H}$ are the single- and two- variables contributions, respectively.

The result (17) is also quite intuitive. The special factors $(x-y)$ and $(\bar{x}-\bar{y})$ are simply $s_{\square}(x \mid y)=(x-y)$ for a single box Young diagram, and its complex conjugate. Moreover, they vanish on $x=y$ or $\bar{x}=\bar{y}$. Thus $\mathcal{H}$ is the part of the correlator which vanishes when both $x=y$ and $\bar{x}=\bar{y}$. The rest, necessarily goes with $(x-y)$ or $(\bar{x}-\bar{y})$, unless it is a constant. In this way, it is also simple to see that the $\mathcal{N}=(4,4)$ Ward identity,

$$
\left[\left(\partial_{x}+\partial_{y}\right) \mathcal{G}\right]_{x=y}=0 ; \quad\left[\left(\partial_{\bar{x}}+\partial_{\bar{y}}\right) \mathcal{G}\right]_{\bar{x}=\bar{y}}=0
$$

is satisfied for any $\mathcal{C}, \mathcal{S}$ and $\mathcal{H}$.

\section{LONG REPRESENTATIONS}

Long superconformal blocks factorize into their bosonic components, i.e., conformal and internal. To see this, take (16) and change basis by considering linear combinations of the form

$$
\frac{1}{2}\left(B_{\left[\kappa_{1}, 1_{1}^{\kappa_{1}^{\prime}-1}\right]} \bar{B}_{\left[\kappa_{2}, 1^{\alpha_{2}^{\prime}-1}\right]} \pm B_{\left[\kappa_{1}, 1^{\kappa_{2}^{\prime}-1}\right]} \bar{B}_{\left[\kappa_{2}, 1^{\kappa_{1}^{\prime}-1}\right]}\right)+\text { c.c. }
$$

This change of basis leads to the general decomposition

$$
\begin{aligned}
\mathcal{H}(x, \bar{x}, y, \bar{y})= & \mathcal{H}^{+}(U, V, \tilde{U}, \tilde{V}) \\
& +(x-\bar{x})(y-\bar{y}) \mathcal{H}^{-}(U, V, \tilde{U}, \tilde{V})
\end{aligned}
$$

where $\mathcal{H}^{ \pm}$will now have a clear relation with bosonic blocks, since they are symmetric in $x, \bar{x}$ and $y, \bar{y}$, and therefore writable as function of $U, V$ and $\tilde{U}, \tilde{V}$. Before giving more details, let us make a first remark: The most general form of a $\mathcal{N}=(4,4)$ correlator, for four half-BPS external particles, is necessarily given by $\mathcal{G}$ in (17), with the splitting of $\mathcal{H}$ as in (20). The dynamical correlator in (2) thus admits two types of kinematics,

kinematics $^{+}=$prefactor $_{p_{3}+p_{4}} \times(x-y)(\bar{x}-\bar{y})$

kinematics ${ }^{-}=(x-\bar{x})(y-\bar{y}) \times$ kinematics $^{+}$

The tree level correlator $\mathcal{A}_{1111}$ of $[23-25,27]$ has $\mathcal{A}_{1111}^{+} \neq 0$, and it was shown that $\mathcal{A}_{1111}^{-}$is absent, correctly. In fact, there is no room for $\mathcal{A}^{-}$, as a polynomial in $y, \bar{y}$, with such a minimal assignment of charges. Assuming a $6 \mathrm{~d}$ conformal symmetry, $\mathcal{A}_{1111}^{+}$is promoted to a generating function for $\mathcal{A}_{\vec{p}}^{+}$through (8), but $\mathcal{A}_{\vec{p}}^{-}$cannot be generated this way.

\section{ON THE $\mathcal{N}=4$ SUPERCONFORMAL BLOCKS}

To analyze $\mathcal{A}_{\vec{p}}^{+}$, we will need an explicit formula for $\mathcal{H}_{\kappa_{1}, \kappa_{2}, \kappa_{1}^{\prime}, \kappa_{2}^{\prime}}^{\text {long }}$. From (11)-(13) we find

$\mathcal{H}^{\text {long }+}=\left(\frac{y \bar{y}}{x \bar{x}}\right)^{\frac{\gamma}{2}(-)^{\kappa_{1}^{\prime}-\kappa_{2}^{\prime}} \mathrm{B}_{\kappa_{1}^{\prime}-\frac{\gamma}{2}, \kappa_{2}^{\prime}-\frac{\gamma}{2}}^{\left(+p_{12},+p_{43}\right)}(y, \bar{y})} \frac{\mathrm{B}_{\kappa_{1}+\frac{\gamma}{2}, \kappa_{2}+\frac{\gamma}{2}}^{\left(-p_{12},-p_{43}\right)}(x, \bar{x})}{U}$

with the bosonic (and normalized) block [40]

$$
\mathrm{B}_{k_{1} k_{2}}^{(a, b)}(z, \bar{z})=\frac{z h_{k_{1}}^{\left(\frac{a}{2}, \frac{b}{2}, 0\right)}(z) \bar{z} h_{k_{2}}^{\left(\frac{a}{2}, \frac{b}{2}, 0\right)}(\bar{z})+\text { c.c. }}{2\left(1+\delta_{k_{1} k_{2}}\right)}
$$

Note that prefactor $\times$ (22) does not depend on $\gamma$ anymore, since it can be absorbed into the $S O(2,2) \times$ $S O(4)$ quantum numbers of $\mathcal{H}^{\text {long+ }}$, which are

$$
\begin{aligned}
1+\frac{\tau}{2} & =\frac{\gamma}{2}+\kappa_{2} ; & l & =\kappa_{1}-\kappa_{2} \geq 0 \\
\frac{b}{2} & =\frac{\gamma}{2}-\kappa_{1}^{\prime} ; & a & =\kappa_{1}^{\prime}-\kappa_{2}^{\prime} \geq 0
\end{aligned}
$$

where recall that $\kappa_{i=1,2} \geq 1$ by construction.

A nice surprise, perhaps expected from the fact that $(1,1) \times \overline{(1,1)}$ might contain a $(2,2)$ factor, comes with $\mathcal{H}^{\text {long- }}$ : This combination of hypergeometrics has bosonic quantum numbers identified as 


$$
\begin{aligned}
1+\frac{\tau}{2} & =\frac{\gamma}{2}+\kappa_{2} ; & l+1 & =\kappa_{1}-\kappa_{2} \geq 0 \\
\frac{b}{2} & =\frac{\gamma}{2}-\kappa_{1}^{\prime} ; & a+1 & =\kappa_{1}^{\prime}-\kappa_{2}^{\prime} \geq 0
\end{aligned}
$$

where this time $\kappa_{1}^{\prime}-\kappa_{2}^{\prime} \geq 1$, by antisymmetry, and it is precisely the same combination of hypergeometrics showing up in the long sector of $\mathcal{N}=4$ SYM [39]. In the latter, the Young diagrams are $(2,2)$ and come in differently, for example $\kappa_{2}=2+\frac{\tau-\gamma}{2}$ and $\kappa_{1}-\kappa_{2}=l$. However, it is simple to see that the arguments of the ${ }_{2} F_{1}$, will coincide [41]. Thus the set of $\mathcal{H}^{\text {long- }}$ is spanned by the same bosonic blocks that appear in $\mathcal{N}=4 \mathrm{SYM}$ in $4 \mathrm{~d}$.

\section{LONG TWO-PARTICLES OPERATORS WITH FLAVOR}

The two-particle operators we want to study are long operators exchanged in $\mathcal{M}^{\mathbb{S}}$ and $\mathcal{M}^{\mathbb{A}}$, and have the schematic form

$$
\mathcal{O}_{(r s)}^{+f}=\mathcal{P}_{I J}^{+f}\left[\mathcal{O}_{r}^{I} \partial^{l} \square^{\frac{1}{2}(\tau-r-s)} \mathcal{O}_{s}^{J}\right]
$$

where $\mathcal{P}^{+f}$ is an appropriate projection for the flavor indexes. At leading order, 4pt diagrams in supergravity are those of a generalized disconnected free theory, and by simple counting the two-particle operators above are degenerate. For given $S O(2,2)$ quantum numbers $\tau, l$, and $S O(4)$ representation $R=[a b]$, the number of degenerate states is nicely organized into a rectangle

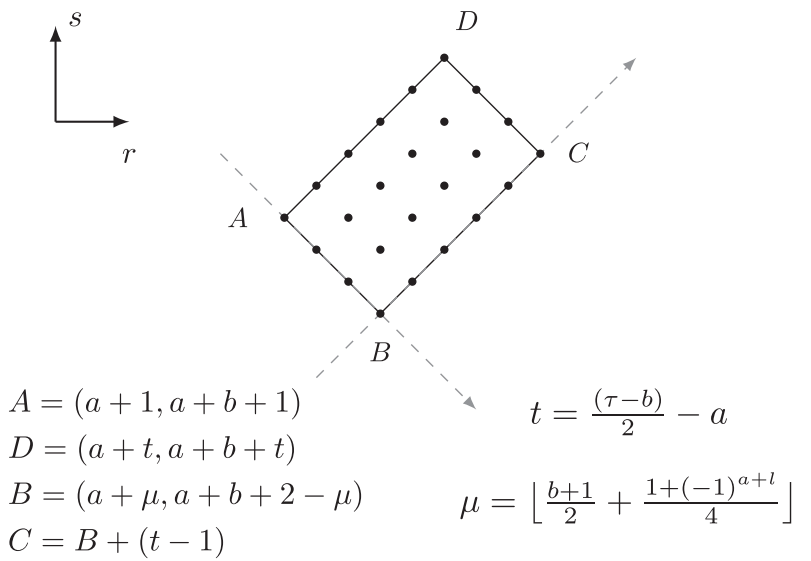

This rectangle $R_{\tau, l,[a b]}$ is analogous to the one in [14].

Since the two-particle operators (26) are long, i.e., nonprotected, they are expected to acquire an anomalous dimension of order $1 / N$, i.e., a binding energy in the gravity picture. We will compute their anomalous dimensions from the consistency of the OPE decomposition of the $4 \mathrm{pt}$ functions. We will only need two sets of matrix equations, given below in (29) and (30), involving threepoint couplings.
Leading three-point couplings of the $\mathcal{O}_{(r s)}^{+f}$ with the external single-particle operators fit into a matrix

$$
\mathbf{C}_{(p q),(r s)} \quad(p q),(r s) \in R_{\tau, l,[a b]}
$$

where $(p q)$ refers to the pair of external operators $\mathcal{O}_{p} \mathcal{O}_{q}$, while $(r s)$ labels the two-particle operator.

The $\mathbf{C}_{(p q),(r s)}$ are found from disconnected Witten diagrams. These exist only for $\left\langle\mathcal{O}_{p} \mathcal{O}_{q} \mathcal{O}_{p} \mathcal{O}_{q}\right\rangle$. In the following we will denote by $L(p q p q)$ the coefficients of their superconformal block decomposition in the long sector. Note that because of the degeneracy, the three-point couplings are not one-to-one with $L(p q p q)$, rather the data is organized in the form of matrix multiplication,

$\mathbf{C}_{\left(p_{1} p_{2}\right),(r s)} \cdot \mathbf{C}_{(r s),\left(p_{3} p_{4}\right)}^{T}=\delta_{p_{1} p_{3}} \delta_{p_{2} p_{4}}\left[\mathbf{L}\left(p_{1} p_{2} p_{3} p_{4}\right)\right]$

as can be quickly derived from the OPE. There are $\mathbf{L}^{ \pm}$ because we have two structure. Then, on a given $R_{\tau, l,[a b]}$ the matrices $\mathbf{L}^{ \pm}$are diagonal.

The anomalous dimensions of the $\mathcal{O}_{(r s)}^{+}$enter the leading logarithmic discontinuity of the tree level correlators, through the equations

$$
\mathbf{C}_{\left(p_{1} p_{2}\right),(r s)} \cdot \boldsymbol{\eta}^{+} \cdot \mathbf{C}_{(r s),\left(p_{3} p_{4}\right)}^{T}=\mathbf{M}\left(p_{1} p_{2} p_{3} p_{4}\right)
$$

where $\boldsymbol{\eta}^{+}$is diagonal and $\mathbf{M}$ is defined from the decomposition

$$
\begin{aligned}
\left.\mathcal{A}_{\vec{p}}^{+}\right|_{\log U}= & \sum_{a, b} \frac{\mathrm{B}_{-\frac{b}{2},-\frac{b}{2}-a}^{\left(+p_{12},+p_{43}\right)}(y, \bar{y})}{\tilde{U}^{1-\frac{p_{4}+p_{3}}{2}}} \\
& \times\left[\sum_{\tau, l} \mathbf{M}_{\tau, l, a, b}(\vec{p}) \frac{\mathrm{B}_{1+\frac{\tau}{2}+l, 1+\frac{\tau}{2}}^{\left(-p_{12},-p_{43}\right)}(x, \bar{x})}{U^{1+\frac{p_{4}+p_{3}}{2}}}\right]
\end{aligned}
$$

Some useful comments on this block decomposition are in order $^{3}$

(i) For a given correlator $\tau \geq \max \left(p_{1}+p_{2}, p_{3}+p_{4}\right)$. It simple to see this inequality from Mellin space: Assume first $p_{1}+p_{2} \leq p_{3}+p_{4}$ then $c_{s} \leq 0$ and $s=0$ is the first double pole in (4). Since the leading term from the right-hand side (rhs) of (31) goes like $U^{\frac{\tau-p_{3}+p_{4}}{2}}$ we find $\tau=p_{3}+p_{4}$. Similarly, if $c_{s} \geq 0$ then $s=c_{s} \geq 0$ is the first double pole, and by the same argument we now find the leading twist to be $\tau=c_{s}-p_{3}-p_{4}=p_{1}+p_{2}$.

(ii) The matrix $\mathbf{M}$ is full, i.e., all entries are non trivial.

${ }^{3}$ Note that can assume without loss of generality that $\vec{p}$ is such that $p_{43} \geq p_{21} \geq 0$. This means for example that the diagram prefactor $\gamma=p_{4}-p_{3}$ exchanges a dimension $=p_{43}$ half-BPS operator, generalizing the identity exchange for equal charges. 
At this point, normalizing $\mathbf{M}$ with the square root of $\mathbf{L}^{+}$ from the left and the right yields an unmixing matrix whose eigenvalues are the anomalous dimensions, and the corresponding eigenvectors, the three-point couplings normalized. This is the same procedure adapted from [11,14], and we will give some explicit example in the next sections.

\section{UNMIXING EXAMPLES}

It is useful, before presenting general formulas, to exemplify the mixing problem in a few cases of interest. We will discuss first the symmetric flavor channel $f=\mathbb{S}$, and comment on $f=\mathbb{A}$ at the end, since the two will be related by a transformation.

The simplest rep we can study is $\mathfrak{R}=[00]$. The first case we can look at is the unique two-particle operator at $\tau=2$ and even spin $l=0,2, \ldots 2 \mathbb{N}$. This case has no mixing,

$\mathbf{L}_{\tau=2, l,[00]}=\frac{(l+1) !^{2}}{(2 l+2) !} \times 2 ; \quad \mathbf{M}_{\tau=4, l,[00]}=\frac{(l+1) !^{2}}{(2 l+2) !} \times 4$

are $1 \times 1$ matrices. The first mixing problem is at $\tau=4$, where we find two even spin operators. The corresponding data is

$$
\begin{aligned}
& \mathbf{L}_{\tau=4, l,[00]}=\frac{(l+2) !^{2}}{(2 l+4) !}\left[\begin{array}{cc}
\frac{2}{3} & 0 \\
0 & \frac{1}{6}(l+1)(l+4)
\end{array}\right] \\
& \mathbf{M}_{\tau=4, l,[00]}=\frac{(l+2) !^{2}}{(2 l+4) !}\left[\begin{array}{cc}
+4 & -4 \\
-4 & 10+5 l+l^{2}
\end{array}\right]
\end{aligned}
$$

Anomalous dimensions and three point functions are obtained by rewriting the mixing problem as an eigenvalue problem. In particular, for $\tau=4$ we find

$$
\mathbf{L}^{-\frac{1}{2}} \cdot \mathbf{M}_{\tau=4, l,[00]} \cdot \mathbf{L}^{-\frac{1}{2}}=\mathbf{c} \cdot\left[\begin{array}{cc}
-\frac{6(l+3)}{l+1} & 0 \\
0 & -\frac{6(l+2)}{l+4} 0
\end{array}\right] \cdot \mathbf{c}^{T}
$$

with

$$
\mathbf{c}_{\tau=4, l,[00]}=\left[\begin{array}{cc}
\sqrt{\frac{l+1}{2 l+5}} & +\sqrt{\frac{l+4}{2 l+5}} \\
-\sqrt{\frac{l+4}{2 l+5}} & \sqrt{\frac{l+1}{2 l+5}}
\end{array}\right]
$$

an orthogonal matrix. The columns of this matrix $\mathbf{c}$ are the eigenvectors of the mixing problem. The leftmost eigenvector corresponds to the most negative anomalous dimension. We will label it with the leftmost corner of $R_{\tau, l,[00]}$. As the value of the anomalous dimension increases we move to the right of this corner. The rep [00] has no degeneracy thus $R_{\tau, l,[00]}$ is simply a line, and all anomalous dimensions are labeled uniquely.

Next, let us consider the rep $\mathfrak{R}=[10]$. This is analogous to $[00]$, but for the fact that only odd spins contribute $l=1,3, \ldots, 2 \mathbb{N}+1$. The first two cases are $\tau=4$ with one operator,

$$
\begin{aligned}
L_{\tau=4, l,[10]} & =\frac{(l+2) !^{2}}{(2 l+4) !} \times \frac{1}{12}\left(24+25 l+5 l^{2}\right) \\
M_{\tau=4, l,[00]} & =\frac{(l+2) !^{2}}{(2 l+4) !} \times 2(l+1)(l+4)
\end{aligned}
$$

and $\tau=6$ with two operators. The mixing problem in this case is found from

$\mathbf{L}_{\tau=6, l,[10]}=\frac{(l+3) !^{2}}{(2 l+6) !} \frac{(120+11(l+7) l)}{40}\left[\begin{array}{cc}1 & 0 \\ 0 & \frac{1}{9}(l+1)(l+6)\end{array}\right]$

$\mathbf{M}_{\tau=6, l,[10]}=\left(\mathbf{L}^{\frac{1}{2}} \mathbf{c}\right) \cdot\left[\begin{array}{cc}-\frac{120(l+4)(l+5)}{(120+11(l+7) l)} & 0 \\ 0 & -\frac{6(l+2)(l+3)}{(120+11(l+7) l)}\end{array}\right] \cdot\left(\mathbf{L}^{\frac{1}{2}} \mathbf{c}\right)^{T}$

where

$$
\mathbf{c}_{\tau=6, l,[10]}=\left[\begin{array}{cc}
\sqrt{\frac{l+1}{2 l+7}} & +\sqrt{\frac{l+6}{2 l+7}} \\
-\sqrt{\frac{l+6}{2 l+7}} & \sqrt{\frac{l+1}{2 l+7}}
\end{array}\right]
$$

Comparing with the $2 \times 2$ case from the $\mathfrak{R}=[00]$, the free theory matrix $\mathbf{L}_{[10]}$ has some overall nonfactorizable term. By construction, this only affects the anomalous dimensions. The matrix $\mathbf{c}$ in (37) has instead the same features as in (34). Quite remarkably, the matrix in (37) is the same matrix that appears in the unmixing problem of [see [11], Eq. (138)] in $\mathcal{N}=4$ SYM in $4 d .{ }^{4}$ We can help our intuition here by using Young diagrams. In fact only Young diagrams that produce an $a \neq 0$ in $[a b]$ can be antisymmetrized in (19), to yield the same as an $\mathcal{N}=4$ SYM block, as follows from the discussion below (25). The case $a=1$, $b=0$ is the first case we find: We have originally two $(1,1)$ Young diagrams $\underline{\kappa}_{i=1,2}$ with two rows, i.e., $\underline{\kappa}_{i}=\left[\kappa_{i 1}, \kappa_{i 2}\right]$, where

$$
\kappa_{i 2} \in\{\varnothing, \square\} ; \quad \kappa_{i 1} \geq 1
$$

Therefore we find three " + " blocks from $\varnothing \times \varnothing, \square \times \square$, and $\varnothing \times \square+\square \times \varnothing$ and a single "-" block from $\varnothing \times \square-\square \times \varnothing$. As we discussed, the latter is the same as the $\mathcal{N}=4$ block that was used in [[11], Eq. (138)].

\footnotetext{
${ }^{4}$ To see this in formulas, we just need to realize that the values of the spins in (37) are assumed to be odd, while those considered in [[11], Eq. (138)] were even.
} 
We close the list of examples by illustrating a mixing problem with partial degeneracy. The simplest case of partial degeneracy appears in $\mathfrak{R}=[02]$, even spins $l=$ $0,2, \ldots 2 \mathbb{N}$ and $\tau=6 .^{5}$ Note that $\tau=6$ is not the first available twist in the rep, which is instead $\tau=4$, but rather the next one. In fact, $R_{\tau=6, l,[02]}$ consists of four points, and is a full rectangle, instead $R_{\tau=4, l,[02]}$ only covers a $-45^{\circ}$ oriented edge. The CFT data we are interested in to see the partial degeneracy is

$$
\mathbf{L}_{\tau=6, l,[02]}=\frac{(l+3) !(l+4) !}{(2 l+6) !} \times \operatorname{diag}\left[\frac{2(l+4)}{15}, \frac{3(l+1)(l+4)(l+6)}{160}, \frac{3(l+3)}{5}, \frac{(l+1)(l+3)(l+6)}{10}\right]
$$

and

$$
\mathbf{M}_{\tau=6, l,[02]}=\frac{(l+3) !(l+4) !}{(2 l+6) !} \times\left[\begin{array}{cccc}
\frac{2\left(15+4 L^{2}\right)}{3(-1+2 L)} & -\frac{23+4 L^{2}}{2(-1+2 L)} & 8 & -8 \\
-\frac{23+4 L^{2}}{2(-1+2 L)} & \frac{1715+40 L^{2}+48 L^{4}}{128(-1+2 L)} & -8 & \frac{55+4 L^{2}}{8} \\
8 & -8 & \frac{5+12 L^{2}}{1+2 L} & -\frac{2\left(7+4 L^{2}\right)}{1+2 L} \\
-8 & \frac{55+4 L^{2}}{8} & -\frac{2\left(7+4 L^{2}\right)}{1+2 L} & \frac{265-40 L^{2}+16 L^{4}}{8(1+2 L)}
\end{array}\right]
$$

where we introduced $l=L-\frac{7}{2}$ just to simplify expressions. The eigenvalues are

$$
\frac{1}{10} \boldsymbol{\eta}_{\tau=6, l,[02]}=-\operatorname{diag}\left[\frac{(l+5)}{l+1}, \frac{(l+2)(l+5)}{(l+3)(l+4)}, \frac{(l+2)(l+5)}{(l+3)(l+4)}, \frac{(l+2)}{(l+6)}\right]
$$

The leftmost root is indexed, in $R_{\tau=6, l,[02]}$, by the leftmost corner at $(r s)=(13)$, then the two (degenerate) middle ones are indexed by $(r s)=(24),(22)$, and the rightmost by the rightmost corner at $(r s)=(33)$.

Let us now comment that when we consider $f=\mathbb{A}$, what happens is that for given $\Re$ even and odd spin sectors are exchanged with respect to $f=\mathbb{S}$, but otherwise the mixing problem is the same. For this reason, we will restrict to $f=\mathbb{S}$ without loss of generality.

Our next task is to find general formulas for the mixing problem. We will begin with free disconnected theory, and then move to the anomalous dimensions.

\section{CAUCHY IDENTITY FOR DISCONNECTED GRAPHS}

The superconformal block decomposition of a free theory graphs, with $g_{i j}$ propagators connecting the various operators, can be done by decomposing the corresponding $\mathcal{C}, \mathcal{S}, \mathcal{H}^{ \pm}$, for levels: first $\mathcal{C}$, then $\mathcal{S}$ and finally $\mathcal{H}^{ \pm}$, paying attention to include at each level the contributions from the previous ones. For a disconnected Witten diagram, thus a disconnected graph, the first superconformal block contributing in each level has $\tau=p+q$, since this equals the

\footnotetext{
${ }^{5}$ This rep also allows for odd spins, but there is no degeneracy for odd spins and we will not discuss it.
}

total number of bridges in the graph going from $\mathcal{O}_{p} \mathcal{O}_{q}$ to $\mathcal{O}_{p} \mathcal{O}_{q}$, i.e., $\gamma=p+q$.

A more illuminating way of performing the same decomposition is to use a Cauchy identity, as shown in [39]. If $q>p$, there is one disconnected graph and the relevant identity is quite compact,

$$
\begin{aligned}
1 & =\left.\sum_{\underline{\kappa}=\left[\kappa, 1^{\kappa^{\prime}-1}\right]} A_{\gamma, \kappa+\frac{\gamma}{2}, \kappa^{\prime}-\frac{\gamma}{2}} F_{\gamma, \underline{\kappa}}^{(\alpha, \beta)}(x, y)\right|_{\gamma=p+q} \\
A_{\gamma, \kappa, \kappa^{\prime}} & =\frac{\Gamma\left[\kappa \pm \frac{q-p}{2}\right] \Gamma\left[2-2 \kappa^{\prime}\right] \Gamma\left[\kappa \pm \frac{\gamma}{2}\right]^{ \pm 1}}{\Gamma[2 \kappa-1] \Gamma\left[1-\kappa^{\prime} \pm \frac{q-p}{2}\right] \Gamma\left[\frac{\gamma+1 \mp 1}{2} \pm \kappa^{\prime}\right]} \frac{(-)^{\kappa+\kappa^{\prime}}}{\Delta_{\kappa, \kappa^{\prime}}^{(2)}}
\end{aligned}
$$

with $\Delta_{\kappa \kappa^{\prime}}^{(2)}=\left(\kappa^{\prime}-\kappa\right)\left(\kappa+\kappa^{\prime}-1\right)$. The mechanism behind (41) is quite neat: Recall that $F_{\gamma, \underline{0}}=1+\cdots$ for $\beta \geq 1$ thus the r.h.s. of (41) is nontrivial precisely because it has to compensate this half-BPS contribution.

From the $(1,1)$ Cauchy identity, we obtain the decomposition for the corresponding $\mathrm{AdS}_{3}$ graph by taking $1 \times \overline{1}$, expanding the sums and recognising, out of the product, the relevant superconformal blocks. In the case $p=q$ there is an additional graph contributing with $[(1-y) /(1-x)]^{\gamma / 2}$. Since $p_{12}=p_{43}=0$, this is related by crossing to (41), and we find again $(-)^{|\underline{\mid}|} A_{\gamma, k, \kappa^{\prime}}$. All together, the decomposition of disconnected graphs relative to $\mathcal{H}^{\text {long } \pm}$, is 


$$
\frac{L_{\tau, l,[a b]}^{ \pm}(p q p q)}{\left(1+\delta_{p q}\right)}=\frac{C_{\kappa, \kappa^{\prime}} C_{\bar{\kappa}, \bar{\kappa}^{\prime}} \pm C_{\kappa, \bar{\kappa}^{\prime}} C_{\bar{\kappa}, \kappa^{\prime}}}{p q}
$$

where $C_{\kappa, \kappa^{\prime}}=A_{p+q, \kappa+\frac{\gamma}{2}, \kappa^{\prime}-\frac{\gamma}{2}}$, with the labels identified as in (24) for $L^{+}$and as in (25) for $L^{-}$. As we pointed out already, it should be the case that $L^{-}$is the same as in $\mathcal{N}=4$ SYM. Indeed,

$L^{-} \propto \operatorname{Gammas} \times \frac{(l+1)(a+1)(a+b+2)(\tau+l+2)}{\delta^{(8)}}$

where $\delta^{(8)}=\delta_{\kappa \kappa^{\prime}}^{(2)} \delta_{\bar{\kappa} \bar{k}^{\prime}}^{(2)} \delta_{\kappa \bar{k}^{\prime}}^{(2)} \delta_{\bar{\kappa} \kappa^{\prime}}^{(2)}$ and $\delta_{\kappa \kappa^{\prime}}^{(2)}=\Delta_{\kappa+\frac{\gamma}{2}, \kappa^{\prime}-\frac{\gamma}{2}}^{(2)}$. This in fact is the fully factorized formula obtained in $[14,19,42]$, which we now discover to be secretly a 2-by-2 determinant.

\section{ANOMALOUS DIMENSIONS}

Knowing the matrix $\mathbf{M}$, we can determine the anomalous dimensions. Unfortunately it is hard to find a closed form expression for $\mathbf{M}$, but working out many cases we have found that the anomalous dimensions of $\mathcal{O}_{(p q)}^{+f}$ with $f=\mathbb{S}$ are consistent with the formula ${ }^{6}$

$$
\begin{aligned}
\eta_{\tau, l,[a b]}^{+}(r s) & =-\frac{2}{N} \frac{\delta^{(8)}}{\delta_{\kappa \kappa^{\prime}}^{(2)} \delta_{\bar{\kappa} \bar{\kappa}^{\prime}}^{(2)}+\delta_{\kappa \bar{\kappa}^{\prime}}^{(2)} \delta_{\bar{\kappa} \prime^{\prime}}^{(2)}} \frac{1}{\left(\ell_{6 d}+1\right)_{2}} \\
\ell_{6 d} & =l+2 r-a-1-\frac{1+(-1)^{a+l}}{2}
\end{aligned}
$$

which is the main result of our paper.

The $\eta_{\tau, l,[a b]}^{+}(r s)$ are simple rational functions of the quantum numbers, and very reminiscent of the tree level anomalous dimensions for $\mathrm{AdS}_{5} \times S^{5}$ two-particle operators found in [14]. Quoting from there

$$
\begin{aligned}
& \eta_{\tau, l,[a b a]}^{\mathrm{AdS}_{5} \times S^{5}}(r s)=-\frac{2}{N^{2}} \delta^{(8)} \frac{1}{\left(\ell_{10 d}+1\right)_{6}} \\
& \ell_{10 d}=l+2 r-a-2-\frac{1+(-1)^{a+l}}{2}
\end{aligned}
$$

Looking at (44), we see now that $\operatorname{AdS}_{5} \times S^{5}$ tree level correlators were such that their decomposition in blocks simplified $\delta_{\kappa \kappa^{\prime}}^{(2)} \delta_{\bar{\kappa} \bar{k}^{\prime}}^{(2)}-\delta_{\bar{k} \bar{k}^{\prime}}^{(2)} \delta_{\bar{\kappa} \kappa^{\prime}}^{(2)}$ in the numerator of $L^{-}$. In fact, we also discover that this combination is fully factorized. On the other hand, $\delta_{\kappa \kappa^{\prime}}^{(2)} \delta_{\bar{\kappa} \bar{\kappa}^{\prime}}^{(2)}+\delta_{\kappa \bar{\kappa}^{\prime}}^{(2)} \delta_{\bar{\kappa} \kappa^{\prime}}^{(2)}$ factorizes only for $a=0$, otherwise it remains generic, as we saw in the unmixing examples in $\mathfrak{R}=[10]$. Thus only when $\mathfrak{R}=[0 b]$ we find

\footnotetext{
${ }^{6}$ The factor $\mathbb{D}$ introduced in (1) is the part $\delta$ dependent of (44).
}

the simplification $\delta^{(8)} /\left(\delta_{\kappa \kappa^{\prime}}^{(2)} \delta_{\bar{\kappa} \bar{\kappa}^{\prime}}^{(2)}+\delta_{\kappa \bar{\kappa}^{\prime}}^{(2)} \delta_{\bar{\kappa} \kappa^{\prime}}^{(2)}\right)=\delta^{(4)}$ which is itself fully factorized.

The partial degeneracy of the $\eta_{\tau, l, a, b}^{+}(r s)$ comes from the fact that they only depend on $r$, rather than $(r s)$, thus twoparticle operators whose labels are on the same vertical axis in $R_{\tau, l,[a b]}$ have degenerate tree level anomalous dimension. This indeed is the same mechanism at work in (46) for $\mathrm{AdS}_{5} \times S^{5}$. In comparison, the large spin behavior goes like $-1 / l^{0}$, and $-1 / l^{2}$, in two, and four dimensions, respectively.

Finally, for given $\mathfrak{R}=[a b]$, when $b$ is even, the set of anomalous dimensions is invariant under $l \rightarrow-l-\tau-1$ in a given spin sector, and when $b$ is odd, the set of anomalous dimensions in the even spin sector is exchanged with those of the odd spin sector. This can be checked explicitly in the unmixing examples, and is just reciprocity symmetry. It was $l \rightarrow-l-\tau-3$ in $\operatorname{AdS}_{5} \times S^{5}$.

\section{HIDDEN SYMMETRY}

We will now comment on the tree level amplitudes for $\mathrm{AdS}_{3} \times S^{3}$ (and $\mathrm{AdS}_{5} \times S^{5}$ ) discussed (and mentioned) above, from the point of view of a higher dimensional conformal symmetry. It will be convenient to introduce $\theta=2,4$ to parametrize $\operatorname{AdS}_{\theta+1} \times S^{\theta+1}$, then the tree level amplitudes for generic charges $\vec{p}$ descend from a single generating function, which is $\mathcal{A}_{\frac{\theta}{2} \frac{\theta}{2} \frac{\theta}{2}}$ after a replacement of the cross ratios [19]. This specific $\mathcal{A}_{\frac{\theta}{2} \frac{\theta \theta}{2} \frac{\theta}{2}}$ is singlet under the sphere, and therefore the cross ratios of $S O(\theta, 2)$ can be replaced with those of $S O(2 \theta+2,2)$ canonically, e.g., using the $\operatorname{AdS} \times S$ Witten diagrams of [18]. In Mellin space, this operation is the covariantization $\mathcal{M}_{\vec{p}}=$ $\mathcal{M}_{1111}(\mathbf{s}, \mathbf{t})$ for $\mathrm{AdS}_{3} \times S^{3}$ and $\mathcal{M}_{\vec{p}}=\mathcal{M}_{2222}(\mathbf{s}, \mathbf{t})$ for $\mathrm{AdS}_{5} \times S^{5}[33]$.

The parameter $\theta$ plays the role of dimensions in two ways. We have $\theta=d=2,4$ for the spacetime dimension of the CFT dual to the $\mathrm{AdS}_{d+1} \times S^{d+1}$ gravity theory. Then we have $\theta=\frac{D-2}{2}$ where $D$ is the dimension of the flat background, i.e., $D=2 d+2=2 \theta+2$, which is simply $\mathrm{AdS}_{d+1} \times S^{d+1}$ in conformally flat coordinates. This suggests that $\mathcal{A}_{\frac{\theta}{2} \frac{\theta \theta}{2} \frac{\theta}{2}}$ should then have a natural decomposition not only in long superconformal blocks for the corresponding SCFT, but also in $S O(2 \theta+2,2)$ conformal blocks at the unitarity bound. Indeed, we find that

$$
\left.\mathcal{A}_{\frac{\theta}{2} \frac{\theta \theta}{2} \frac{\theta}{2}}\right|_{\log U}=\sum_{\ell} \frac{\theta \Gamma[\ell+\theta]^{2}}{\Gamma[2 \ell+2 \theta-1]} \frac{{ }_{2} F_{1}[\theta+\ell, \theta+\ell ; 2 \theta+2 \ell ; P]}{U^{\theta}}
$$

where ${ }_{2} F_{1}[\theta+\ell, \ldots]$ is a single normalized block in which we understand the ${ }_{2} F_{1}$ as a power series with the replacement $z^{n} \rightarrow P_{[\theta+\ell+n, \theta]}(x, \bar{x} ; \theta)$, and $P(; \theta)$ being the twovariables Jack polynomial. A compact way of writing this polynomial is 
$\frac{(\theta)_{k}}{k !} \frac{P_{[\theta+k, \theta]}(x, \bar{x} ; \theta)}{U^{\theta}}=e^{-k \varphi} \sum_{j=0}^{k} \frac{(\theta)_{j}(\theta)_{k-j}}{j !(k-j) !} e^{i(k-2 j) \phi}$

where $x=e^{-\varphi+i \phi}$ as in [43,44] (and $k=\ell+n$ ).

Quite remarkably the $S O(2 \theta+2,2)$ decomposition in (48) only runs over a single sum. In fact, the second row of $P(; \theta)$ does not grow. To recover the usual double expansion over twist and spin of $S O(d, 2)$ we need to recognize that within a $P_{\underline{\underline{K}}}(; \theta)$ there are various $P_{\underline{\underline{\nu}}}\left(; \theta^{\prime}\right)$ where $\theta^{\prime}=$ $(d-2) / 2$ and $\bar{d}=2,4$ [40], for $\theta=2,4$ respectively. In fact,

$$
\begin{aligned}
P_{\underline{\underline{\kappa}}}(\theta)= & \sum_{m \geq 0} P_{\left[\kappa_{1}-m, \kappa_{2}+m\right]}\left(\theta^{\prime}\right) \times \frac{(-)^{m}}{m !} \\
& \times\left[\frac{\left(\theta-\theta^{\prime}+1-m\right)_{m} \times\left(\kappa_{-}+1-2 m\right)_{2 m}}{\left(\theta+\kappa_{-}-m\right)_{m}\left(\theta^{\prime}+\kappa_{-}-m\right)_{m}}\right]
\end{aligned}
$$

where $\kappa_{-}=\kappa_{1}-\kappa_{2}$, and $\left(\kappa_{-}+1-2 m\right)_{2 m}$ truncates the sum. Changing from $P_{\underline{\nu}}\left(; \theta^{\prime}\right)$ to the bosonic blocks [40] gives the usual type of expansion.

From the generating function, we obtain $\mathcal{A}_{\vec{p}}=\hat{\mathcal{D}}_{\vec{p}}\left[U^{\theta} \mathcal{A}_{\frac{\theta}{2} \frac{\theta}{2} \frac{\theta}{2} 2}\right]$, where

$$
\hat{\mathcal{D}}_{\vec{p}}=\frac{1}{(U \tilde{U})^{\frac{\theta}{2}}} \sum_{\tilde{s}, \tilde{t}}\left(\frac{\tilde{U}}{U}\right)^{\tilde{s}+\frac{\theta}{2}}\left(\frac{\tilde{V}}{V}\right)^{\tilde{t}} \hat{\mathcal{D}}_{\vec{p},(\tilde{s}, \tilde{t})}^{(0,0,0)} \hat{\mathcal{D}}_{\vec{p},(\tilde{s}, \tilde{t})}^{\left(c_{s}, c_{t}, c_{u}\right)}
$$

is a differential operator. As in [33] we can find its explicit expression,

$$
\begin{aligned}
\hat{\mathcal{D}}_{\tilde{p},(\tilde{s}, \tilde{t})}^{(a, b, c)}= & \frac{\left(U \partial_{U}+1-\theta-\tilde{s}-a\right)_{\tilde{s}+a}}{(-)^{a}(\tilde{s}+a) !} \\
& \times \frac{\left(V \partial_{V}+1-\tilde{t}-b\right)_{\tilde{t}+b}}{(-)^{b}(\tilde{t}+b) !} \frac{\left(U \partial_{U}+V \partial_{V}\right)_{\tilde{u}+c}}{(\tilde{u}+c) !}
\end{aligned}
$$

Understanding the action of $(52)$ on $P(; \theta)$, for example in (49), might help finding an explicit formula for the threepoint couplings. Indeed, by acting with $\hat{\mathcal{D}}_{\vec{p}}$ on a single ${ }_{2} F_{1}[\theta+\ell, \ldots ; P]$ and summing, yields by construction the mixing matrix, say on a $R_{\tau, l,[a b]} \otimes R_{\tau, l,[a b]}$ for reference. As pointed out in [19], this computation actually gives the mixing matrix as $\oplus_{r}\left(\eta \times \mathfrak{P}_{r}\right)$ where $\mathfrak{P}_{r}$ is a projector built out of the three-point couplings. In particular, these projectors descend from the ${ }_{2} F_{1}[\theta+\ell, \ldots ; P]$ and there are as many projectors as values of $r$ in $R_{\tau, l,[a b]}$. However, only when there is no residual degeneracy the projector is one-dimensional.

Regarding the lift of the $\mathrm{AdS}_{3} \times S^{3}$ partial degeneracy, let us comment that from the $\operatorname{AdS}_{d+1} \times S^{d+1}$ VirasoroShapiro action postulated in [18], and specialized to our $d=2$ case, we have found evidence that a mechanism analogous to that discovered in [16,17], will fix uniquely the three-point couplings.

\section{OUTLOOK}

The D1-D5 system has various tractable corners (see for example [45-49]), and most notably, the weak coupling regime has a (world sheet) WZW description. But the 2d theory at the boundary of $\mathrm{AdS}_{3} \times S^{3}$ with pure RR flux, whose $4 \mathrm{pt}$ correlators we have studied in this paper, is strongly coupled. The bootstrap approach is therefore quite natural in this case, since it does not rely on having a weakly coupled Lagrangian description. In fact, the many clues of hidden simplicity that we have encountered encourage the idea that our bootstrap program can tackle quantitatively this strongly coupled regime, offering new dynamical insights, beyond tree level. The clues we have found are neatly summarized by the form of the anomalous dimensions in (44), and nicely accompanied by the structure of the generating function (48), which provides the seed for the leading logarithmic discontinuity at any loop order. As in $\mathrm{AdS}_{5} \times S^{5}$ we can now start computing one-loop correlators, and we will do so elsewhere.

On top of the above findings, we noted that the tree level dynamics (of tensor multiplets) on $\mathrm{AdS}_{3} \times S^{3}$ and that on $\mathrm{AdS}_{5} \times S^{5}$ are aligned in many details. Worth mentioning is the fact that the chiral correlators, defined as [40], $\mathcal{G}^{\text {chiral }}:=\left.\mathcal{G}\right|_{\bar{y}=\bar{x}}=\mathcal{C}+(x-y) \mathcal{S}(x, y)$, are actually equal in both theories, which suggests that the $(1,1)$ superconformal blocks "factorize" both theories, and tempts the idea that maybe there is a mechanism to understand the correlator, beyond the protected sector [50], which still uses a Chern-Simons/WZW correspondence.

More speculatively, it is also the case that when integrability techniques can be applied, some correlators [51] have a free fermion description [52,53], therefore it would be very interesting to understand whether the same is true for the AdS $\times S$ correlators we studied in this paper.

Finally, the possibility of having $\mathcal{A}_{\vec{p}}^{-} \neq 0$ with $p_{i}>1$ remains open.

\section{ACKNOWLEDGMENTS}

We thank J. M. Drummond, P. Heslop and P. Vieira for many discussions, and especially S. Giusto, R. Russo and the authors of [54] for providing important feedback and sharing their draft with us. M. S. thanks D. Bufalini, H. Paul and S. Rawash for discussion. F. A. is partially supported by the ERC-STG Grant No. 637844- HBQFTNCER, and M. S. by a Mayflower studentship from the University of Southampton. 
[1] J. M. Maldacena, Adv. Theor. Math. Phys. 2, 231 (1998).

[2] L. Rastelli and X. Zhou, Phys. Rev. Lett. 118, 091602 (2017); J. High Energy Phys. 04 (2018) 014.

[3] L. F. Alday and A. Bissi, Phys. Rev. Lett. 119, 171601 (2017).

[4] F. Aprile, J. M. Drummond, P. Heslop, and H. Paul, J. High Energy Phys. 01 (2018) 035.

[5] F. Aprile, J. M. Drummond, P. Heslop, and H. Paul, J. High Energy Phys. 05 (2018) 056.

[6] L. F. Alday, A. Bissi, and E. Perlmutter, J. High Energy Phys. 06 (2019) 010.

[7] J. M. Drummond and H. Paul, J. High Energy Phys. 03 (2021) 038.

[8] F. Aprile, J. Drummond, P. Heslop, and H. Paul, J. High Energy Phys. 03 (2020) 190.

[9] A. Bissi, G. Fardelli, and A. Georgoudis, Phys. Rev. D 104, L041901 (2021); arXiv:2010.12557.

[10] J. M. Drummond, R. Glew, and H. Paul, arXiv:2008.01109.

[11] F. Aprile, J. M. Drummond, P. Heslop, and H. Paul, J. High Energy Phys. 02 (2018) 133.

[12] S. M. Chester, J. High Energy Phys. 04 (2020) 193.

[13] D. J. Binder, S. M. Chester, S. S. Pufu, and Y. Wang, J. High Energy Phys. 12 (2019) 119.

[14] F. Aprile, J. Drummond, P. Heslop, and H. Paul, Phys. Rev. D 98, 126008 (2018).

[15] J. M. Drummond, D. Nandan, H. Paul, and K. S. Rigatos, J. High Energy Phys. 12 (2019) 173.

[16] J. M. Drummond, H. Paul, and M. Santagata, arXiv:2004.07282 [Phys. Rev. D (to be published)].

[17] F. Aprile, J. M. Drummond, H. Paul, and M. Santagata, J. High Energy Phys. 11 (2021) 109.

[18] T. Abl, P. Heslop, and A. E. Lipstein, J. High Energy Phys. 04 (2021) 237.

[19] S. Caron-Huot and A. K. Trinh, J. High Energy Phys. 01 (2019) 196.

[20] J. de Boer, Nucl. Phys. B548, 139 (1999).

[21] N. Berkovits, C. Vafa, and E. Witten, J. High Energy Phys. 03 (1999) 018.

[22] M. Taylor, J. High Energy Phys. 06 (2008) 010.

[23] S. Giusto, R. Russo, and C. Wen, J. High Energy Phys. 03 (2019) 096.

[24] S. Giusto, R. Russo, A. Tyukov, and C. Wen, J. High Energy Phys. 09 (2019) 030.

[25] S. Giusto, R. Russo, A. Tyukov, and C. Wen, Eur. Phys. J. C 80, 736 (2020).

[26] S. Giusto, M. R. R. Hughes, and R. Russo, J. High Energy Phys. 11 (2020) 018.
[27] L. Rastelli, K. Roumpedakis, and X. Zhou, J. High Energy Phys. 10 (2019) 140.

[28] L. J. Romans, Nucl. Phys. B276, 71 (1986).

[29] S. Deger, A. Kaya, E. Sezgin, and P. Sundell, Nucl. Phys. B536, 110 (1998).

[30] M. Mihailescu, J. High Energy Phys. 02 (2000) 007.

[31] G. Arutyunov, A. Pankiewicz, and S. Theisen, Phys. Rev. D 63, 044024 (2001).

[32] We use the same conventions as in [33].

[33] F. Aprile and P. Vieira, J. High Energy Phys. 12 (2020) 206.

[34] J. Penedones, J. High Energy Phys. 03 (2011) 025; A. L. Fitzpatrick, J. Kaplan, J. Penedones, S. Raju, and B. C. van Rees, J. High Energy Phys. 11 (2011) 095.

[35] Y. H. Lin, S. H. Shao, Y. Wang, and X. Yin, J. High Energy Phys. 12 (2015) 142.

[36] M. Heydeman, J. H. Schwarz, C. Wen, and S. Q. Zhang, Phys. Rev. Lett. 122, 111604 (2019).

[37] S. Giombi, R. Roiban, and A. A. Tseytlin, Nucl. Phys. B922, 499 (2017).

[38] L. Córdova and P. Vieira, J. High Energy Phys. 12 (2018) 063.

[39] R. Doobary and P. Heslop, J. High Energy Phys. 12 (2015) 159.

[40] F. A. Dolan and H. Osborn, Nucl. Phys. B678, 491 (2004); Ann. Phys. (Amsterdam) 321, 581 (2006).

[41] See formulas (74) in section III.1.1. of [11].

[42] E.g., formula (A.33) of [17] has our same conventions.

[43] T. Bargheer, F. Coronado, and P. Vieira, arXiv:1909.04077.

[44] A. V. Belitsky and G. P. Korchemsky, J. High Energy Phys. 05 (2020) 070.

[45] J. M. Maldacena and H. Ooguri, J. Math. Phys. (N.Y.) 42, 2929 (2001); Phys. Rev. D 65, 106006 (2002).

[46] M. R. Gaberdiel and I. Kirsch, J. High Energy Phys. 04 (2007) 050.

[47] A. Pakman and A. Sever, Phys. Lett. B 652, 60 (2007).

[48] L. Eberhardt, M. R. Gaberdiel, and R. Gopakumar, J. High Energy Phys. 02 (2020) 136.

[49] B. Eden, D. 1. Plat, and A. Sfondrini, J. High Energy Phys. 08 (2021) 049.

[50] F. Bonetti and L. Rastelli, J. High Energy Phys. 08 (2018) 098.

[51] F. Coronado, J. High Energy Phys. 01 (2019) 056; Phys. Rev. Lett. 124, 171601 (2020).

[52] I. Kostov, V. B. Petkova, and D. Serban, Phys. Rev. Lett. 122, 231601 (2019).

[53] I. Kostov and V. B. Petkova, J. High Energy Phys. 06 (2021) 098.

[54] N. Ceplak, S. Giusto, M. R. R. Hughes, and R. Russo, arXiv:2105.04670. 